\title{
Lower-Outer Quadrant of the Breast
}

National Cancer Institute

\section{Source}

National Cancer Institute. Lower-Outer Quadrant of the Breast. NCI Thesaurus. Code C12304.

The quarter of the breast which is inferior and lateral. 